\title{
COMUNICAÇÃO
}

\section{RELAÇÃO ENTRE TRICOMAS FOLIARES DE GENÓTIPOS DE FEIJOEIRO COMUM, Phaseolus vulgaris L. E RESISTÊNCIA A Diabrotica speciosa Germar, 1824 (Coleoptera: Chrysomelidae)}

\author{
Relationship between foliar trichomes of common bean genotypes, Phaseolus vulgaris L., \\ and resistance to Diabrotica speciosa Germar, 1824(Coleoptera: Chrysomelidae)
}

\author{
Maria José Fachini de Oliveira Paron¹, Fernando Mesquita Lara²
}

\begin{abstract}
RESUMO
Considerando que a pubescência foliar pode afetar a preferência alimentar do inseto, com o presente estudo objetivou-se verificar a influência da pilosidade dos folíolos na resistência de genótipos de feijoeiro a $D$. speciosa. $\mathrm{O}$ experimento foi conduzido em delineamento experimental inteiramente casualizado, com seis tratamentos e oito repetições. Os tratamentos foram seis genótipos de feijoeiro: Emgopa Ouro e IAPAR 57, do centro de domesticação Mesoamericano (MA) e Jalo Precoce, Goiano Precoce, PR 10595142 e PR 10595146 do centro de domesticação Andino (AN). Cada parcela foi constituída por um vaso com 1,2 Kg de solo e uma planta encerrada em um telado individual. No 25을 dia após o plantio, em cada telado foram liberados dez adultos de $D$. speciosa, após jejum de 24 horas, durante 72 horas. Os danos sofridos foram estimados de acordo com a área consumida em cada genótipo. Os tricomas das folhas dos genótipos foram contados usando microscópio estereoscópico (40x), com uma escala micrométrica sobre $1 \mathrm{~cm}^{2}$ de área da superfície abaxial (AB) e adaxial (AD), contando-se os tricomas unciformes e aciculares presentes em cinco folhas de cada genótipo. O genótipo IAPAR 57 (MA) foi o preferido no teste de alimentação, enquanto os genótipos andinos foram menos consumidos por adultos de $D$. speciosa. Os genótipos apresentaram variabilidade quanto ao número de tricomas, tanto aciculares quanto unciformes, em ambas as superfícies, abaxial e adaxial. Os genótipos andinos não diferiram quanto ao número de tricomas unciformes na superfície adaxial. O somatório do número de tricomas aciculares e unciformes na superfície abaxial dos genótipos sugerem relação inversa entre esta característica e a área consumida por $D$. speciosa.
\end{abstract}

Termos para indexação: Pubescência, preferência alimentar, resistência de plantas, feijão, Fabaceae.

\begin{abstract}
Considering that foliar pubescence may affect insect feeding preference on plant genotypes, the purpose of this study was to evaluate the influence of leaflet pubescence on resistance of common bean genotypes to $D$. speciosa. The experiments were conducted in a completely randomised design, with six treatments and eight replicates. The treatments were six common bean genotypes, Emgopa Ouro and IAPAR 57, from Mesoamerican domestication center (MA), Jalo Precoce, Goiano Precoce, PR 10595142 and PR 10595146 from Andean domestication center (AN). Each replicate consisted of one pot with $1.2 \mathrm{~kg}$ soil and a plant placed in a individual cage. The genotypes were submitted to feeding test 25 days after planting, when ten adults of $D$. speciosa, starved for 24 hours, were released for 72 hours in each cage. Damage was estimated by area of foliage consumed on each genotype. Trichomes on both the abaxial (AB) and adaxial (AD) leaf surfaces were counted using a stereomicroscope $(40 \mathrm{X})$, on $1 \mathrm{~cm}^{2}$ area, with the hooked and straight trichomes being counted on five leaflets/genotype. The MA genotype IAPAR 57 was the most preferred on feeding test, while the AN genotypes were less eaten by Dispeciosa. The genotypes showed variability aceading to density of trichomes on both surfaces. Andean genotypes showed no difference in unciform trichome densities on $\mathrm{AD}$ surface. The total number of straight and hooked trichomes on $\mathrm{AB}$ leaf surface suggests inverse relation between this characteristic and the area consumed by Diabrotica speciosa
\end{abstract}

Index terms: Pubescence, feeding preference, host plant resistance, common bean, Fabaceae.

\section{(Recebido para publicação em 17 de dezembro de 2003 e aprovado em 18 de março de 2005)}

De acordo com Heyer et al. (1986), a pubescência dos folíolos de feijoeiro, Phaseolus vulgaris L., pode influenciar a aceitação do alimento por insetos crisomelídeos. Nessa leguminosa, os tricomas foliares variam em tamanho e densidade, de acordo com o centro de origem: nos genótipos do centro de domesticação andino eles são densos e longos, enquanto que nos do centro de domesticação Mesoamericano, são esparsos e curtos (VIEIRA et al., 1999).

A importância de tricomas foliares na resistência de $P$. vulgaris foi observada para algumas espécies de

\footnotetext{
1 Engenheira Agrônoma, Dr., Departamento Defesa Vegetal - Universidade Estadual Paulista/ UNESP - Faculdade de Ciências Agronômicas, Fazenda Experimental Lageado - Caixa Postal 237 - 18603-970 - Botucatu, SP.

${ }^{2}$ Professor Titular, Departamento de Fitossanidade - Universidade Estadual Paulista/ UNESP - Faculdade de Ciências Agrárias e Veterinárias -

Via de Acesso Prof. Paulo Donato Castellane s/n - 14.884-900 - Jaboticabal, SP.
} 
insetos-pragas. Mizukoshi \& Kakizaki (1995) relataram que Aphis solani ficaram fisicamente aprisionados nos tricomas unciformes, morrendo por inanição. Para a cigarrinha Empoasca fabae, a presença de tricomas unciformes é um dos fatores que conferem resistência a alguns genótipos de feijoeiro, chegando a aumentar a mortalidade das ninfas de quatro a dez vezes (PILLEMER \& TINGEY, 1976), embora esse mesmo tipo de tricoma não tenha sido eficiente contra Empoasca kraemeri (KORNEGAY \& CARDONA, 1991). Lyman et al. (1981) estudaram a resistência de variedades de Phaseolus lunatus frente a E. kraemeri e encontraram correlação negativa entre o dano e número de tricomas por $\mathrm{cm}^{2}$. No caso da larva minadora Liriomyza trifolii, a captura de adultos esteve correlacionada positivamente com a densidade de tricomas unciformes (QUIRING et al., 1992).

Conduziu-se este trabalho com o objetivo de verificar a influência da pilosidade de folíolos de genótipos de feijoeiro na resistência à vaquinha, Diabrotica speciosa (Coleoptera: Chrysomelidae), em plantas crescidas sob telados individuais.

O experimento foi conduzido em delineamento experimental inteiramente casualizado, com seis tratamentos e oito repetições. Os tratamentos foram seis genótipos de feijoeiro: cultivares Emgopa Ouro e IAPAR 57, do centro de domesticação Mesoamericano (MA), cultivares Jalo Precoce e Goiano Precoce e linhagens PR 10595142 e PR 10595146, do centro de domesticação Andino (AN). Cada parcela foi constituída por um vaso com $1,2 \mathrm{~kg}$ de solo e uma planta encerrada em um telado. No 25 dia após o plantio, em cada telado foram liberados dez adultos, após jejum por 24 horas, durante 72 horas. Em cada 10 adultos introduzidos por telado, 8 foram provenientes de criação em dieta natural (ÁVILA et al., 2000) e 2 coletados no campo, nas culturas de soja e abóbora. Após aquele período as folhas foram coletadas, etiquetadas e guardadas na forma de excicatas para avaliação das áreas consumidas em integrador eletrônico de superfície (LICOR-5000) (PARON \& LARA, 2001).

A quantidade de tricomas epidérmicos nas duas faces dos folíolos dos genótipos foi determinada por contagem sob microscópio estereoscópico (40x), com uma escala micrométrica sobre $1 \mathrm{~cm}^{2}$ de área, contando-se os tricomas unciformes e aciculares presentes em cinco folíolos de cada genótipo.

Os dados foram tabulados, submetidos à análise de variância (teste F), teste de médias (Tukey, 5\%) e equação de regressão, utilizando-se o programa estatístico ESTAT .
Os tricomas aciculares apresentaram densidade de zero a 41,00 $\mathrm{cm}^{-2}$ na superfície abaxial (AB), sendo os valores nulos correspondentes aos genótipos Emgopa 201 Ouro, Jalo Precoce e Goiano Precoce, os quais diferiram dos mais pilosos PR 10595142 e PR 10595146, mas não diferiram do Mesoamericano IAPAR 57. Na face adaxial (AD) a variação foi de 2,00 a 7,80 $\mathrm{cm}^{-2}$ com Jalo Precoce, PR $95105142 \mathrm{e}$ Goiano Precoce sendo os mais glabros, diferindo de PR 10595146 e IAPAR 57, mais pilosos, mas não diferindo de Emgopa 201 Ouro (Tabela 1).

Os tricomas unciformes foram mais numerosos, alcançando valores médios entre 14,60 e $105,00 \mathrm{~cm}^{-2}$ na face abaxial e entre zero e $14,40 \mathrm{~cm}^{-2}$ na face adaxial. $\mathrm{Na}$ face abaxial os genótipos Goiano Precoce e Jalo Precoce destacaram-se como os mais pubescentes, sendo que Goiano Precoce não diferiu de PR 95105146, que por sua vez não diferiu de PR 95105142; este último não diferiu de Emgopa 201 Ouro e IAPAR 57. Na face adaxial os genótipos andinos não diferiram quanto ao número de tricomas unciformes, Goiano Precoce e Jalo Precoce com superaram os genótipos Mesoamericanos; Emgopa 201 Ouro não apresentou tricomas deste tipo na superfície adaxial.

Os valores máximos de densidade de tricomas aciculares na superfície abaxial encontrados ficaram aquém dos obtidos em P. vulgaris por Heyer et al. (1986) (até $162,50 \mathrm{~cm}^{-2}$ ), Peña et al. (1992) (até $1400 \mathrm{~cm}^{-2}$ ) e Dahlin et al. (1992) (até $386 \mathrm{~cm}^{-2}$ ). Os unciformes também tiveram densidades médias menores do que o relatado na literatura para a espécie, tais como os encontrados por Heyer et al. (1986) (até $177 \mathrm{~cm}^{-2}$ ), Peña et al. (1992) (até $2000 \mathrm{~cm}^{-2}$ ) e Dahlin et al. (1992) (até $307 \mathrm{~cm}^{-2}$ ). Tal variação pode ocorrer devido ao genótipo em estudo, pois segundo Dahlin et al. (1992), cultivares de $P$. vulgaris mostram grande variação em tipo e densidade de tricomas. Além disso, Lara (1991) menciona que a densidade de tricomas e outras formações da epiderme das plantas estão sujeitas às variações ambientais, como umidade, luz, temperatura e características do solo. O feijoeiro apresenta maior número de tricomas em ambiente seco, pois, entre outras funções, a pubescência aumenta a condutividade térmica da superfície da folha, aumentando a perda de calor por convecção, tendo importância para plantas em ambientes quentes (DAHLIN et al., 1992). Em feijoeiro, Silva et al. (1988) encontraram maiores densidades de tricomas unciformes abaxiais em níveis mais altos de radiação do que sob sombreamento (consorciado com milho). Como as plantas do presente estudo cresceram sob condições de casa-de- 
vegetação, onde a luminosidade é inferior à encontrada em condições naturais, devido à refração da luz solar no teto, tal condição pode ter resultado em menor densidade de tricomas, pois, segundo Smith \& Nobel (1978), folhas sob baixa radiação apresentam menor pubescência, e conseqüentemente, maior absorção da radiação solar; o grau de plasticidade da pubescência, dependendo da radiação, auxilia o papel adaptativo da folha em alterar a capacidade de absorção. De qualquer forma, a variação na densidade de tricomas observada sugere que as plantas mais pubescentes, tais como as das linhagens PR 95105146 e PR 95105142 (grande número de aciculares) e das cultivares Jalo Precoce e Goiano Precoce (grande número de unciformes) podem dificultar a alimentação de $D$. speciosa, assim como ocorreu no estudo de Heyer et al. (1986), quando testou genótipos mais pubescentes em relação à alimentação de crisomelídeos desfolhadores. Naquele estudo, correlacionaram as baixas taxas de danos por crisomelídeos encontradas na cultivar CC-25-9 R à presença de densa pubescência (grande número de tricomas aciculares longos).

Ao estudar a influência da pubescência de cultivares de trigo na oviposição do crisomelídeo Oulema melanoplus (L.), Schillinger \& Gallun (1968) descobriram efeito deterrente, com fêmeas em fase reprodutiva relutantes para oviposição em cultivares altamente pubescentes, levando a um menor número de ovos/planta e menor número total de ovos colocados. Cultivares com camada densa de tricomas obtiveram menores porcentagens de ovos eclodidos, devido a susceptibilidade à dissecação gerada neste micro habitat.

TABELA 1 - Área foliar consumida por adultos de $D$. speciosa, confinados às plantas em telados individuais e quantidade média de tricomas aciculares e unciformes $\left(\mathrm{n}^{\mathrm{o}} \mathrm{cm}^{-2}\right)$ presentes na superfície abaxial (AB) e adaxial (AD) de folíolos de seis genótipos de feijoeiro. Jaboticabal, SP, 1999.

\begin{tabular}{|c|c|c|c|c|c|}
\hline \multirow{2}{*}{ Genótipos } & \multirow{2}{*}{$\begin{array}{c}\text { Consumo } \\
\left(\mathrm{mm}^{2}\right)^{1 /}\end{array}$} & \multicolumn{2}{|c|}{ Aciculares } & \multicolumn{2}{|c|}{ Unciformes } \\
\hline & & $\mathbf{A B}^{2 /}$ & AD & $\mathbf{A B}$ & AD \\
\hline IAPAR 57 (MA) & 2333,4 a & $7,40 \mathrm{bc}$ & $7,20 \mathrm{ab}$ & $14,60 \mathrm{~d}$ & $3,40 \mathrm{bc}$ \\
\hline Emgopa 201 Ouro (MA) & $994,3 \mathrm{ab}$ & $0,00 \mathrm{c}$ & $3,60 \mathrm{bc}$ & $25,20 \mathrm{~d}$ & $0,00 \mathrm{c}$ \\
\hline PR 95105146 (AN) & $638,9 \mathrm{bc}$ & $41,00 \mathrm{a}$ & $7,80 \mathrm{a}$ & $66,00 \mathrm{bc}$ & $9,40 a b$ \\
\hline Jalo Precoce (AN) & $604,3 \mathrm{bc}$ & $0,00 * \mathrm{c}$ & $2,00 \mathrm{c}$ & $105,00 \mathrm{a}$ & $14,00 \mathrm{a}$ \\
\hline PR 95105142 (AN) & $578,0 \mathrm{bc}$ & $26,00 \mathrm{ab}$ & $2,60 \mathrm{c}$ & $40,00 \mathrm{~cd}$ & $9,60 \mathrm{ab}$ \\
\hline Goiano Precoce (AN) & $197,6 \mathrm{c}$ & $0,00 * \mathrm{c}$ & $3,00 \mathrm{c}$ & $97,40 \mathrm{ab}$ & $14,40 \mathrm{a}$ \\
\hline $\mathrm{CV}(\%)$ & 41,07 & 34,62 & 44,84 & 30,46 & 38,93 \\
\hline
\end{tabular}

\footnotetext{
${ }^{1 /}$ Paron \& Lara (2001).

${ }^{2 /}$ Médias originais. Para análise estatística os dados foram transformados em $(x+0,5)^{0,5}$.

Médias seguidas de mesma letra não diferem entre si pelo Teste de Tukey a 5\% de probabilidade.

* tricomas aciculares presentes somente nas nervuras central e secundária.
} 
Os tricomas unciforme e acicular apresentam diferentes características: o acicular tem forma de agulha e é ereto, medindo de 350 (ORIANI \& LARA, 2000) a 500 $\mathrm{mm}$ (DAHLIN et al., 1992), enquanto o unciforme tem forma de gancho, com ângulo de inserção variável e é menor, com cerca de 75 (ORIANI \& LARA, 2000) a $200 \mathrm{~mm}$ (DAHLIN et al., 1992). Hoxie et al. (1975) estudaram, em 9 linhagens de trigo, os efeitos distintos do tamanho e da densidade de tricomas, classificando-as em seis categorias: poucos e curtos; muitos e curtos; muitíssimos e curtos; poucos e longos; muitos longos e muitíssimos e longos. O número de ovos por planta e a porcentagem de sobrevivência larval de $O$. melanoplus caíram com mudanças na categoria, nesta ordem, e os autores concluíram que, para os danos causados ao trigo por este inseto, o fator de resistência mais importante é o tamanho do tricoma
Na Tabela 1 observa-se que houve maior ataque nos folíolos do genótipo Mesoamericano IAPAR 57, diferindo dos genótipos andinos. Em segundo lugar, a preferência alimentar dos adultos de $D$. speciosa se deu pelo genótipo mesoamericano Emgopa 201 Ouro, que ocupou posição intermediária entre IAPAR 57 e a linhagem andina PR 95105146, não diferindo também dos genótipos PR 95105142 e Jalo Precoce. Considerando que na superfície abaxial foram encontradas as maiores densidades de tricomas, tanto aciculares quanto unciformes, pode-se observar relação inversa entre número de tricomas nessa face e o consumo pelo crisomelídeo. Submetidos os somatórios dos valores encontrados nas superfícies abaxiais dos genótipos à análise de regressão (Figura 1), nota-se esta tendência, com resposta linear. Neste sentido, os resultados obtidos concordam com os encontrados por Heyer et al. (1986) em feijoeiro e por Kanno (1996) em soja.

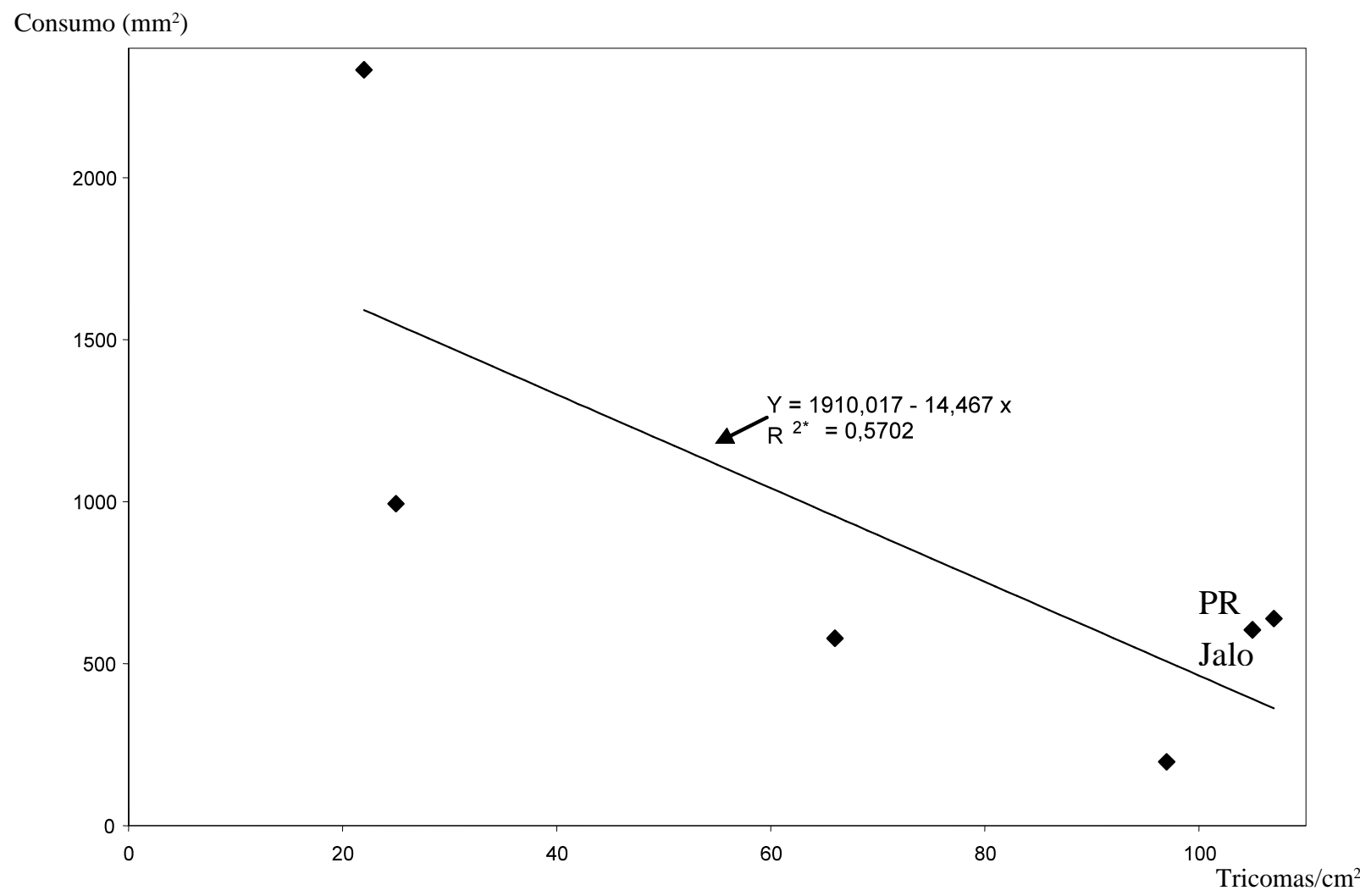

FIGURA 1 - Relação entre número de tricomas (aciculares + unciformes) por $\mathrm{cm}^{2}$ na superfície abaxial de seis genótipos de feijoeiro (x) e área consumida por D. speciosa. Os pontos correspondem aos dados de: IAPAR= IAPAR 57; Emgopa= Emgopa 201 Ouro; PR 146= PR 95105146; Jalo= Jalo Precoce; PR 142= PR 95105142; Goiano= Goiano Precoce. Jaboticabal- SP, 1999. 


\section{AGRADECIMENTOS}

À CAPES pela bolsa de doutorado concedida à primeira autora.

\section{REFERÊNCIAS BIBLIOGRÁFICAS}

ÁVILA, C. J.; TABAI, A. C. P.; PARRA, J. R. P. Comparação de técnicas para criação de Diabrotica speciosa (Germar) (Coleoptera: Chrysomelidae) em dietas natural e artificial. Anais da Sociedade Entomológica do Brasil, Jaboticabal, v. 29, n. 2, p. 257-267, 2000.

DAHLIN, R. M.; BRICK, M. A.; OGG, J. B. Characterization and density of trichomes on three common bean cultivars. Economic Botany, Bronx, v. 46, n. 3, p. 299-304, 1992.

HEYER, W.; CRUZ, B.; CHIANG-LOK, M. L. Comportamiento y preferencia de los adultos de Diabrotica balteata, Andrector ruficornis, Systena basalis (Coleoptera: Chrysomelidae) y Empoasca fabae (Homoptera: Cicadellidae), en frijol. Ciencias de la Agricultura, [S.1.], v. 27, p. 61-76, 1986.

HOXIE, R. P.; WELLSO, S. G; WEBSTER, J. A. Cereal leaf beetle response to wheat trichome lenght and density. Environmental Entomology, College Park, v. 4, n. 3, p. 365370, 1975.

KANNO, H. Role of leaf pubescence in soybean resistance to the false melon beetle, Atrachya menetriesi Faldermann (Coleoptera: Chrysomelidae). Applied Entomology and Zoology, Oxford, v. 31, n. 4, p. 597-603, 1996.

KORNEGAY, J.; CARDONA, C. Breeding for insect resistance in beans. In: SCHOONHOVEN, A. van; VOYEST, O. Crop improvement. Cali: CIAT, 1991. p. 619-648.

LARA, F. M. Princípios de resistência de plantas a insetos. 2. ed. São Paulo: Ícone, 1991. 336 p.

LYMAN, J. M.; CARDONA, C.; GARCIA, J. Estudios sobre la resistencia del frijol lima al Empoasca kraemeri Ross. \& More. Revista Colombiana de Entomologia, [S.1.], v. 7, p. 27-32, 1981.

MIZUKOSHI, T.; KAKIZAKI, M. Influence of trichomes on kidney bean leaves to the development of the foxglove aphid, Aulacorthum solani (Homoptera: Aphididae).
Annual Report of the Society of Plant Protection of North Japan, Tokyo, n. 46, p. 142-146, 1995.

ORIANI, M. A. de G.; LARA, F. M. Oviposition preference of Bemisia tabaci (Genn.) Biotype B (Homoptera: Aleyrodidae) for bean genotypes containing arcelin in the seeds. Anais da Sociedade Entomológica do Brasil, Jaboticabal, v. 29, n. 3, p. 565-572, 2000.

PARON, M. J. F. O.; LARA, F. M. Preferência alimentar de adultos de Diabrotica speciosa (Germar) a genótipos de feijoeiro Phaseolus vulgaris L. Neotropical Entomology, Londrina, v. 30, n. 4, p. 669-674, 2001.

PEÑA, E. A.; PANTOJA, A.; BEAVER, J. Determinación de la pubescencia de cuatro genotipos de habichuela, Phaseolus vulgaris L. Journal of Agriculture of the University of Puerto Rico, [S.1.], v. 76, n. 2, p. 71-82, 1992.

PILLEMER, E. A.; TINGEY, W. M. Hooked trichomes: a physical plant barrier to a major agricultural pest. Science, London, v. 193, p. 482-484, 1976.

QUIRING, D. T.; TIMMINS, P. R.; PARK, S. J. Effect of variations in hooked trichome densities of Phaseolus vulgaris on longevity of Liriomyza trifolii (Diptera: Agromyzidae) adults. Environmental Entomology, College Park, v. 21, n. 6, p. 1357-1361, 1992.

SCHILLINGER, J. A.; GALLUN, R. L. Leaf pubescence of wheat as a deterrent to the cereal leaf beetle, Oulema melanoplus. Annals of the Entomological Society of America, [S.1.], v. 61, n. 4, p. 900-903, 1968.

SILVA, H. T.; RODRIGUES, J. D.; COSTA, J. G. da. Influência do sombreamento causado pelo milho na anatomia da folha de feijoeiro de diferentes hábitos de crescimento: I. estômatos e tricomas. Pesquisa Agropecuária Brasileira, Brasília, v. 23, n. 12, p. 1387-1400, 1988.

SMITH, W. K.; NOBEL, P. S. Influence of irradiation soil, water potential, and leaf temperature on leaf morphology of a desert broad leaf, Encelia farinosa Gray. American Journal of Botany, Columbus, n. 65, p. 429-432, 1978.

VIEIRA, C.; BORÉM, A.; RAMALHO, M. A. P. Melhoramento do feijão. In: BORÉM, A. Melhoramento de espécies cultivadas. Viçosa: UFV, 1999. p. 273-349.

Ciênc. agrotec., Lavras, v. 29, n. 4, p. 894-898, jul./ago., 2005 